\title{
Generalized Field Theoretical Approach to General Relativity and Conserved Quantities in Anti-de Sitter Spacetimes
}

\author{
N. Pinto-Neto* \\ and \\ R. Rodrigues da Silva \\ Centro Brasileiro de Pesquisas Físicas \\ Rua Xavier Sigaud, 150 - Urca \\ 22290-180, Rio de Janeiro, RJ \\ Brazil \\ (November 3, 2018)
}

\begin{abstract}
A new prescription to calculate the total energies and angular momenta of asymptotically $(d+1)$ dimensional anti-de Sitter spacetimes is proposed. The method is based on an extension of the field theoretical approach to General Relativity to the case where there is an effective cosmological constant. A $(d-1)$-form $\Omega$ is exhibited which, when integrated on asymptotic $(d-1)$-dimensional boundary surfaces, yields the values of those conserved quantities. The calculations are gauge independent once asymptotic conditions are not violated. Total energies and angular momenta of some known solutions in four and five dimensions are calculated agreeing with standard results.
\end{abstract}

PACS number(s): 04.20.Cv, 04.20.Ha

\section{INTRODUCTION}

In the last years, a lot of investigations have been done on asymptotically anti-de Sitter (AdS) spacetimes. This interest is widely connected with the development of string theory. For instance, the Maldacena conjecture [1] relates conformal field theories in a $d$-dimensional space with supergravity or string theory on the product of $(d+1)$ dimensional AdS space with a compact manifold, and this result can be used to study the thermodynamics of AdS black holes [2]. One important problem of AdS space concerns the definition of mass and angular momentum of asymptotically AdS spacetimes. Many papers have been written on this subject proposing different ways to calculate such quantities [3 77]. Classically, they all agree in their final results, with the exception of the generalized Komar mass calculated in Ref. [5]. In this paper we will propose a new method to calculate such quantities based on the field theoretical approach to General Relativity (GR) [8 10], which is applicable to either asymptotically flat or asymptotically AdS spacetimes for all $d \geq 2$, and discuss its advantages and drawbacks with respect to the other methods. Our development also clarifies some issues concerning the background metric defined in Refs. [8 10].

In the field theoretical approach to GR, the gravitational field is treated as a spin two field $h^{\mu \nu}$ propagating with self interaction in a background metric $\gamma_{\mu \nu}$, which is usually considered to be Ricci flat. The self interaction and interaction of the spin two field with other matter fields is constructed in such a way that the background metric is never perceived: it appears only through the combination $\sqrt{-g} g^{\mu \nu}=\sqrt{-\gamma}\left(\gamma^{\mu \nu}+h^{\mu \nu}\right)$, and this combination satisfies exactly the Einstein's equations. In terms of $h^{\mu \nu}$ and $\gamma_{\mu \nu}$, the Einstein-Hilbert action and Einstein's equation can be viewed as an action and equations of motion of a spin two field in a Ricci flat background metric. This allows one to define a true energy-momentum tensor of the gravitational field plus matter fields by varying the lagrangian with respect to the background metric. However, the theory in terms of $h^{\mu \nu}$ aquires a gauge freedom which is the manifestation of the invariance of GR under the action of the manifold mapping group (MMG), and the energy momentum tensor calculated in the way explained above is not a gauge invariant quantity. Nevertheless, quantities like the total energy and angular momentum do not suffer from this ambiguity once one specify asymptotic conditions. In fact, some calculations of total energies have been done for asymptotically flat spacetimes [9] agreeing with well known results. Our task is to extend this procedure to asymptotically AdS spacetimes. This extension, however, is not straightforward. This is because of the presence of the cosmological constant in AdS spacetimes. One might think that this should not pose a problem because the formalism developped in Refs. 8 10 are applicable to any matter field, and a cosmological constant can be viewed, for instance, as resulting from the energy density of some scalar field in its ground state. However, when we calculate the total energy of asymptotically AdS spacetimes considering

*e-mail address: nelsonpn@lafex.cbpf.br 
that the background metric is still Ricci flat, we obtain preposterous results, even when we remove the energy of the pure AdS spacetime. The resulting energy does not yield the usual asymptotically flat results when we put the cosmological constant to zero. This result enforces us to take as the background metric some Einstein space satisfying $\stackrel{\circ}{R}_{\mu \nu}=2 \gamma_{\mu \nu} \stackrel{\circ}{\Lambda} /(d-1)$.

In general, the total energy and angular momenta are calculated by the integration of a closed $d$-form $J, d J=0$, on a d-dimensional spacelike volume. The main result of this paper is to exhibit the general expression of a $(d-1)$-form $\Omega$ which fulfills the condition $J=d \Omega$ for the general case of background metrics satisfying $\stackrel{\circ}{R}_{\mu \nu}=2 \gamma_{\mu \nu} \stackrel{\circ}{\Lambda} /(d-1)$, hence proving that $J$ is indeed globally exact in general. This enables one to write those conserved quantities as asymptotic $(d-1)$-dimensional integrals, which rends their calculations feasible, and clarify the conditions for their

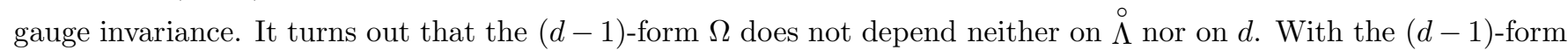
$\Omega$ we have at our disposal an alternative and straightforward method to calculate any conserved quantity of any asymptotically flat or AdS spacetime in any number of dimensions greater than two. We calculated total energies and angular momenta for some known solutions in four and five dimensions using our expression for $\Omega$, and they agree with standard results.

This paper is organized as follows: in the next section we generalize the field theoretical approach to the case of GR with a cosmological constant $\Lambda$ and background metric satisfying $\stackrel{\circ}{R}_{\mu \nu}=2 \gamma_{\mu \nu} \stackrel{\circ}{\Lambda} /(d-1)$ (note that, for the sake of generality, the two cosmological constants may be different). We obtain the total energy-momentum tensor. In section III we write down the conserved current $J$ associated with this total energy-momentum tensor, and we arrive at the $(d-1)$-form $\Omega$. We discuss the conditions for its gauge invariance, and we calculate some conserved quantities of well known examples using this method. We also discuss the necessity of using background metrics satisfying

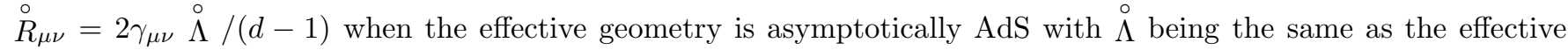
cosmological constant in the effective geometry. We end up in section IV with conclusions and discussions.

\section{THE FIELD THEORETICAL APPROACH FOR GENERAL REALTIVITY WITH A COSMOLOGICAL CONSTANT}

Take the Einstein-Hilbert action depending on a metric $g_{\mu \nu}$ and a symmetric connection $\Gamma_{\mu \nu}^{\alpha}$, together with a cosmological constant $\Lambda$ term, and an action for matter fields without couplings with the connection. In this total action, make the substitutions

$$
\begin{gathered}
\sqrt{-g} g^{\mu \nu}=\sqrt{-\gamma}\left(\gamma^{\mu \nu}+h^{\mu \nu}\right), \\
\Gamma_{\mu \nu}^{\alpha}=C_{\mu \nu}^{\alpha}+K_{\mu \nu}^{\alpha} .
\end{gathered}
$$

where $\gamma_{\mu \nu}$ and $C_{\mu \nu}^{\alpha}$ are the metric and Christoffel symbols, respectively, of a background space satisfying

$$
\stackrel{\circ}{R}_{\mu \nu}=\frac{2 \stackrel{\circ}{\Lambda}}{d-1} \gamma_{\mu \nu}
$$

From now on indices will be raised and lowered by $\gamma_{\mu \nu}$. After discarding surface terms and terms independent on the fields $h^{\mu \nu}$ and $K_{\mu \nu}^{\alpha}$, we obtain:

$$
\begin{aligned}
S= & \frac{1}{16 \pi G} \int\left[\tilde{h}^{\mu \nu}\left(K_{\mu \nu ; \alpha}^{\alpha}-K_{\mu ; \nu}\right)+\left(\tilde{\gamma}^{\mu \nu}+\tilde{h}^{\mu \nu}\right)(K K)_{\mu \nu}\right. \\
& \left.+\frac{2}{d-1} \stackrel{\circ}{\Lambda} \tilde{h}^{\mu \nu} \gamma_{\mu \nu}-2 \Lambda \sqrt{-\bar{g}}\right] d^{d+1} x+\int \mathcal{L}_{M}\left(\Phi_{A}, \bar{g}_{\alpha \beta}\right) d^{d+1} x
\end{aligned}
$$

where

$$
\begin{gathered}
\tilde{\gamma}^{\mu \nu} \equiv \sqrt{-\gamma} \gamma^{\mu \nu}, \tilde{h}^{\mu \nu} \equiv \sqrt{-\gamma} h^{\mu \nu}, \\
K_{\mu} \equiv K_{\alpha \mu}^{\alpha}
\end{gathered}
$$

and 


$$
(K K)_{\mu \nu} \equiv K_{\mu \nu}^{\alpha} K_{\alpha}-K_{\mu \beta}^{\alpha} K_{\nu \alpha}^{\beta} .
$$

The semi-colon (;) represents the covariant derivative with respect to the background connections, and $\Phi_{A}$ represents general matter fields. The terms $\bar{g}_{\alpha \beta}$, which is the inverse of $g^{\mu \nu}$, and $\sqrt{-\bar{g}}$ appearing in the action must be understood as algebraic functions of finite degree polynomials on $h^{\mu \nu}$ and $\gamma_{\mu \nu}$ (see Ref. 11 for their explicit form).

The dynamical equations for the gravitational field can be obtained by performing a Palatini-like variation of the action (2.4 with respect to the fields $\tilde{h}^{\mu \nu}$ and $K_{\mu \nu}^{\alpha}$ independently. We obtain the following equations:

$$
\begin{aligned}
& K_{\mu \nu ; \alpha}^{\alpha}-\frac{1}{2}\left(K_{\mu ; \nu}+K_{\nu ; \mu}\right)+(K K)_{\mu \nu}= \\
& \frac{2}{d-1}\left(\Lambda \bar{g}_{\mu \nu}-\stackrel{\circ}{\Lambda} \gamma_{\mu \nu}\right)+8 \pi G\left(T_{\mu \nu}^{M}-\frac{1}{d-1} T^{M} \gamma_{\mu \nu}\right),
\end{aligned}
$$

and

$$
\tilde{h}_{; \alpha}^{\mu \nu}-\left(\tilde{\gamma}^{\mu \nu}+\tilde{h}^{\mu \nu}\right) K_{\alpha}+\left(\tilde{\gamma}^{\mu \rho}+\tilde{h}^{\mu \rho}\right) K_{\rho \alpha}^{\nu}+\left(\tilde{\gamma}^{\nu \rho}+\tilde{h}^{\nu \rho}\right) K_{\rho \alpha}^{\mu}=0,
$$

where

$$
T_{\mu \nu}^{M}=-\frac{2}{\sqrt{-\gamma}} \frac{\delta \mathcal{L}_{M}}{\delta \gamma^{\mu \nu}}
$$

and $T^{M}$ is its trace. Note that the matter energy-momentum tensor defined above is not the same as the usual matter energy-momentum tensor $t_{\mu \nu}^{M}$ defined using variations of the matter lagrangian with respect to the effective metric $g^{\mu \nu}$. Only the combinations

$$
T_{\mu \nu}^{M}-\frac{1}{d-1} T_{\alpha \beta}^{M} \gamma^{\alpha \beta} \gamma_{\mu \nu}=-2 \frac{\delta \mathcal{L}_{M}}{\delta \tilde{\gamma}^{\mu \nu}}=-2 \frac{\delta \mathcal{L}_{M}}{\delta \tilde{g}^{\mu \nu}}=t_{\mu \nu}^{M}-\frac{1}{d-1} t_{\alpha \beta}^{M} g^{\alpha \beta} g_{\mu \nu}
$$

are equal. Note also that the $T_{\mu \nu}^{M}$ defined above is not covariantly conserved with respect to the background connection.

Eq. (2.9) is equivalent to the determination of the connection $\Gamma_{\mu \nu}^{\alpha}$ in terms of the Christoffel symbols of $g_{\mu \nu}$ while Eq. (2.8) is equivalent to the Einstein's equations when we use Eqs. (2.1,2.2.2.3). After some rearrangements in Eqs. (2.8.2.9) (see Ref. [9] for details), we obtain the following equation:

$$
\begin{aligned}
G_{\mu \nu}^{L}= & -(K K)_{\mu \nu}+\frac{1}{2} \gamma_{\mu \nu}(K K)_{\alpha}^{\alpha}+Q_{\mu \nu ; \alpha}^{\alpha} \\
& +\frac{\Lambda}{d-1}\left(2 \bar{g}_{\mu \nu}-\bar{g}_{\alpha}^{\alpha} \gamma_{\mu \nu}\right)+\stackrel{\circ}{\Lambda} \gamma_{\mu \nu}+8 \pi G T_{\mu \nu}^{M},
\end{aligned}
$$

where

$$
2 G_{\mu \nu}^{L}=\left[\gamma_{\mu \nu} h^{\alpha \beta}+\gamma^{\alpha \beta} h_{\mu \nu}-\delta_{\mu}^{\alpha} h_{\nu}^{\beta}-\delta_{\nu}^{\alpha} h_{\mu}^{\beta}\right]_{; \alpha ; \beta}
$$

and

$$
\begin{aligned}
2 Q_{\mu \nu}^{\alpha} & =-\gamma_{\mu \nu} h^{\rho \sigma} K_{\rho \sigma}^{\alpha}+h_{\mu \nu} K^{\alpha}-h_{\mu}^{\alpha} K_{\nu}-h_{\nu}^{\alpha} K_{\mu}+ \\
& +h_{\mu}^{\rho}\left(K_{\rho \nu}^{\alpha}-K_{\rho \lambda}^{\sigma} \gamma^{\alpha \lambda} \gamma_{\sigma \nu}\right)+h_{\nu}^{\rho}\left(K_{\rho \mu}^{\alpha}-K_{\rho \lambda}^{\sigma} \gamma^{\alpha \lambda} \gamma_{\sigma \mu}\right)+ \\
& +h^{\alpha \rho}\left(K_{\rho \mu}^{\sigma} \gamma_{\sigma \nu}+K_{\rho \nu}^{\sigma} \gamma_{\sigma \mu}\right)
\end{aligned}
$$

The total energy-momentum tensor of the gravitational field plus matter described by the action (2.4) can be calculated as usual by varying the total lagrangian $\mathcal{L}_{T}=\mathcal{L}_{G}+\mathcal{L}_{M}$ with respect to the background metric, yielding

$$
\begin{aligned}
T_{\mu \nu}^{T}= & -\frac{2}{\sqrt{-\gamma}} \frac{\delta \mathcal{L}_{T}}{\delta \gamma^{\mu \nu}}=\frac{1}{8 \pi G}\left[-(K K)_{\mu \nu}+\frac{1}{2} \gamma_{\mu \nu}(K K)_{\alpha}^{\alpha}+Q_{\mu \nu ; \alpha}^{\alpha}\right. \\
& +\frac{\Lambda}{d-1}\left(2 \bar{g}_{\mu \nu}-\bar{g}_{\alpha}^{\alpha} \gamma_{\mu \nu}\right)+\frac{\left.2 \stackrel{\circ}{d-1} h_{\mu \nu}\right]+T_{\mu \nu}^{M} .}{}
\end{aligned}
$$

Equations (2.12) and (2.15) give rise to

$$
G_{\mu \nu}^{L}+\frac{2 \stackrel{\circ}{\Lambda}}{d-1} h_{\mu \nu}=8 \pi G T_{\mu \nu}^{T}+\stackrel{\circ}{\Lambda} \gamma_{\mu \nu}
$$


Due to the Riemannian nature of the background geometry, one can define a new conseved energy-momentum tensor as

$$
T_{\mu \nu}=T_{\mu \nu}^{T}+\frac{\stackrel{\circ}{\Lambda}}{8 \pi G} \gamma_{\mu \nu}
$$

and write Eq. (2.16) as

$$
G_{\mu \nu}^{L}+\frac{2 \stackrel{\circ}{\Lambda}}{d-1} h_{\mu \nu}=8 \pi G T_{\mu \nu}
$$

There are some advantages in adopting $T_{\mu \nu}$ instead of $T_{\mu \nu}^{T}$. For instance, let us assume that the background metric is a solution of the Einstein's equations when the matter fields are in the vacuum state, which is equivalent to say that $h_{\mu \nu}=0$ is a solution of Eqs. (2.8) and (2.9) in vacuum. This is a reasonable assumption, although not mandatory at this moment. In this case, $T_{\mu \nu}\left(h_{\mu \nu}=0\right)$ is identically zero while $T_{\mu \nu}^{T}\left(h_{\mu \nu}=0\right)$ has a residual background term, as can be seen imediately from Eqs. (2.16 2.18). As we will see in the next section, the new $T_{\mu \nu}$ is a renormalized energy-momentum tensor yielding authomatically finite results for conserved quantities.

Note that the covariant divergence of the left-hand-side of Eq. (2.18) is identically zero once the background metric satisfies Eq. (2.3). Hence, the conservation of $T_{\mu \nu}$ can also be obtained as a consequence of the field equations.

As the energy-momentum tensor $T_{\mu \nu}$ is a true tensor, it seems that it does not suffer from the ambiguities which are present in the usual definitions of pseudo-tensors in GR. However, the field theoretical approach to GR has an invariance under true gauge transformations that comes from the invariance of GR under the action of the manifold mapping group. The $T_{\mu \nu}$ above defined is not a gauge invariant quantity, as it will now be seen.

The coordinate transformation invariance of GR is translated to invariance under gauge transformations on $h^{\mu \nu}$ and $K_{\mu \nu}^{\alpha}$ in the field theoretical approach in the following way: consider the infinitesimal coordinate transformation $x^{\alpha}=x^{\alpha}+\xi^{\alpha}(x)$, which changes the functional form of $\tilde{g}^{\mu \nu}$ as

$$
\tilde{g}^{\prime \mu \nu}(x)=\tilde{g}^{\mu \nu}(x)+£_{\xi}^{(1)} \tilde{g}^{\mu \nu}(x),
$$

where the Lie derivative $£_{\xi}^{(1)} \tilde{g}^{\mu \nu}$ is given by

$$
£_{\xi}^{(1)} \tilde{g}^{\mu \nu}(x)=-\tilde{g}_{, \lambda}^{\mu \nu} \xi^{\lambda}+\tilde{g}^{\lambda \mu} \xi_{, \lambda}^{\nu}+\tilde{g}^{\lambda \nu} \xi_{, \lambda}^{\mu}-\tilde{g}^{\mu \nu} \xi_{, \sigma}^{\sigma} .
$$

In the case of a finite transformation, the change in $\tilde{g}^{\mu \nu}$ is given by

$$
\tilde{g}^{\prime \mu \nu}(x)=\tilde{g}^{\mu \nu}(x)+\sum_{k=1}^{\infty} \frac{1}{k !} £_{\xi}^{(k)} \tilde{g}^{\mu \nu}
$$

where $£_{\xi}^{(k)}$ is the Lie derivative of order $k$ defined as

$$
£_{\xi}^{(k)}=£_{\xi}^{(1)}\left[£_{\xi}^{(k-1)}\right]
$$

Substituting in 2.21) the definition (2.1) we get

$$
\tilde{g}^{\prime \mu \nu}(x)=\tilde{\gamma}^{\mu \nu}(x)+\tilde{h}^{\mu \nu}(x)+\sum_{k=1}^{\infty} \frac{1}{k !} £_{\xi}^{(k)}\left(\tilde{\gamma}^{\mu \nu}+\tilde{h}^{\mu \nu}\right)
$$

The transformed metric density can be decomposed in two distinct ways:

$$
\tilde{g}^{\prime \mu \nu}(x)=\tilde{\gamma}^{\mu \nu}(x)+\tilde{h}^{\prime \mu \nu}(x)
$$

and

$$
\tilde{g}^{\prime \mu \nu}(x)=\tilde{\gamma}^{* \mu \nu}(x)+\tilde{h}^{* \mu \nu}(x),
$$

where, by comparison with $(2.23)$ one gets 


$$
\begin{gathered}
\tilde{h}^{\prime \mu \nu}(x)=\tilde{h}^{\mu \nu}(x)+\sum_{k=1}^{\infty} \frac{1}{k !} £_{\xi}^{(k)}\left(\tilde{\gamma}^{\mu \nu}+\tilde{h}^{\mu \nu}\right), \\
\tilde{h}^{* \mu \nu}(x)=\tilde{h}^{\mu \nu}(x)+\sum_{k=1}^{\infty} \frac{1}{k !} £_{\xi}^{(k)} \tilde{h}^{\mu \nu},
\end{gathered}
$$

and

$$
\tilde{\gamma}^{* \mu \nu}(x)=\tilde{\gamma}^{\mu \nu}(x)+\sum_{k=1}^{\infty} \frac{1}{k !} £_{\xi}^{(k)} \tilde{\gamma}^{\mu \nu} .
$$

Analogously, the gauge transformation corresponding to Eq. (2.24) for the field $K_{\mu \nu}^{\alpha}(x)$ is

$$
K_{\mu \nu}^{\prime \alpha}(x)=K_{\mu \nu}^{\alpha}(x)+\sum_{k=1}^{\infty} \frac{1}{k !} £_{\xi}^{(k)}\left(C_{\mu \nu}^{\alpha}+K_{\mu \nu}^{\alpha}\right)
$$

while for the case corresponding to the transformation (2.25) reads

$$
K_{\mu \nu}^{* \alpha}(x)=K_{\mu \nu}^{\alpha}(x)+\sum_{k=1}^{\infty} \frac{1}{k !} £_{\xi}^{(k)} K_{\mu \nu}^{\alpha} .
$$

Eqs. (2.25), 2.27),(2.28) and (2.30) represent the usual transformations on tensorial fields resulting from a general mapping of the manifold on which they are defined. Hence, all tensors in the manifold, in particular the energymomentum tensor defined above, will transform in the usual homogeneous way:

$$
T^{\prime \mu \nu}(x)=T^{\mu \nu}(x)+\sum_{k=1}^{\infty} \frac{1}{k !} £_{\xi}^{(k)} T^{\mu \nu}(x)
$$

The situation is completely different for the case of Eqs. 2.24), (2.26) and (2.29). Those transformations act only on the fields $\tilde{h}^{\mu \nu}$ and $K_{\mu \nu}^{\alpha}$, letting invariant the background metric $\gamma^{\mu \nu}(x)$. In this sense, one can interpret them as true gauge transformations. It can be shown that the dynamical equations for the gravitational field (2.12) transform as a combination of themselves under the transformations (2.26) and (2.29), supposing that the background space satisfies $\stackrel{\circ}{R}_{\mu \nu}=2 \stackrel{\circ}{\Lambda} \gamma_{\mu \nu} /(d-1)$. The new field $\tilde{h}^{\prime \mu \nu}(x)$ is also a solution of the field equations, and it corresponds to the same physical field as $\tilde{h}^{\mu \nu}(x)$. In this case, the tensors do not transform in the usual way (2.31) but contains extra inhomogeneous terms which brings the possibility of annulling them. In this case, the energy-momentum tensor, for example, transforms according to:

$$
\begin{aligned}
& T_{\mu \nu}\left(h^{\prime}, K^{\prime}\right)=T_{\mu \nu}(h, K)+\frac{1}{16 \pi G}\left\{\hat{G}_{\mu \nu}^{L}\left[\frac{1}{\sqrt{-\gamma}} \sum_{k=1}^{\infty} \frac{1}{k !} £_{\xi}^{(k)}\left(\tilde{\gamma}^{\alpha \beta}+\tilde{h}^{\alpha \beta}\right)\right]\right.
\end{aligned}
$$

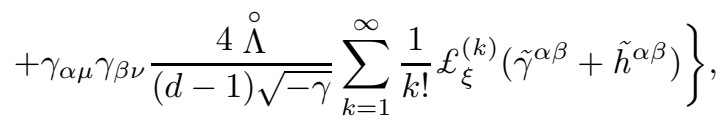

where $\hat{G}_{\mu \nu}^{L}$ is the operator which when acting on $h^{\alpha \beta}$ yields expression (2.13). Hence, it is always possible to find gauge transformations (2.26) and (2.29) which makes the energy-momentum tensor defined previously to be null. This is the analogue in the field theoretical approach to what happens with the pseudotensors in GR. Note that the new energy-momentum tensor in Eq. (2.32) is also covariantly conserved due to the properties of $G_{\mu \nu}^{L}$.

We have now completed our generalization of the field theoretical approach for the case when the background metric satisfies Eq. (2.3), which includes the Ricci flat case when we make $\stackrel{\AA}{\Lambda}=0$. Although the energy-momentum tensor defined above suffers from gauge ambiguities, the total energy or angular momentum of a gravitational field does not. As we will see in the next section, total conserved quantities are given by integration of a $d$-form on a $d$-dimensional hypersurface, which can be reduced to an integration of a $(d-1)$-form on the $(d-1)$-dimensional boundary of this hypersurface at the asymptotic limit. As one would like to preserve the asymptotic structure of the gravitational field, the gauge vectors $\xi^{\alpha}$ must satisfy boundary conditions at the asymptotic $(d-1)$-dimensional surfaces. They must tend to AdS Killing vectors at spatial infinity (see Ref. 《t for details), and the true gauge transformations reduce to the identity at the boundaries. Hence, as the total conserved quantities can be calculated by integration on the asymptotic boundary, they are gauge independent. 


\section{THE $(D-1)$-FORM WHICH GIVES TOTAL CONSERVED QUANTITIES OF ASYMPTOTICALLY ANTI-DE SITTER SPACETIMES}

Consider the energy-momentum tensor $T_{\mu \nu}$ defined in Eqs. (2.15) and (2.17), and a Killing form $\xi_{\nu}$ of the background metric. Construct, as usual, the current

$$
J^{\mu}=T^{\mu \nu} \xi_{\nu}
$$

and define the $d$-form

$$
J=\frac{1}{d !} J^{\mu} \eta_{\mu \alpha_{1} \ldots \alpha_{d}} d x^{\alpha_{1}} \wedge \ldots \wedge d x^{\alpha_{d}}
$$

where $\eta_{\mu \alpha_{1} \ldots \alpha_{d}}=\sqrt{-\gamma} \epsilon_{\mu \alpha_{1} \ldots \alpha_{d}}$ and $\epsilon_{\mu \alpha_{1} \ldots \alpha_{d}}$ is the $(d+1)$-dimensional (metric independent) completely antisymmetric object.

Due to energy-momentum conservation together with the Killing equation, it follows that $J_{; \mu}^{\mu}=0$, which is equivalent to $d J=0$, i.e., $J$ is a closed $d$-form. Hence,

$$
\int_{M} d J=\int_{\partial M} J=0
$$

where $M$ is a $(d+1)$-dimensional spacetime volume. If the boundary $\partial M$ of $M$ is constituted of two $d$-dimensional spacelike hypersurfaces $\Sigma_{1}$ and $\Sigma_{2}$ labelled by the time parameters $t_{1}$ and $t_{2}$, respectively, and a $d$ dimensional timelike hypersurface $B$ at spatial infinity, and supposing that the fields and Killing vectors are such that $J$ is zero at $B$ (which is the case for the non-radiating and asymptotically AdS gravitational fields we will study in this paper), then Eq. (3.3) reduces to

$$
\int_{\Sigma_{2}} J-\int_{\Sigma_{1}} J=0
$$

and $\int_{\Sigma} J$ is a conserved quantity. If the Killing vector field is timelike or associated with some rotational symmetry, then we will have a conserved total energy or a conserved total angular momentum, respectively. Looking at the equations of motion (2.18) and the definition of $G_{\mu \nu}^{L}$ in Eq. (2.13), we can write the conserved current $J^{\mu}$ on shell as:

$$
J^{\mu}=\frac{1}{16 \pi G}\left[\left(\gamma^{\mu \nu} h^{\alpha \beta}+\gamma^{\alpha \beta} h^{\mu \nu}-\gamma^{\alpha \mu} h^{\beta \nu}-\gamma^{\alpha \nu} h^{\beta \mu}\right)_{; \alpha ; \beta} \xi_{\nu}+\frac{4 \stackrel{\circ}{\Lambda}}{d-1} h^{\mu \nu} \xi_{\nu}\right] .
$$

Using the fact that the background metric satisfies $\stackrel{\circ}{R}_{\mu \nu}=2 \gamma_{\mu \nu} \stackrel{\circ}{\Lambda} /(d-1)$, the last term in the right-hand-side of the above equation can be written in a suitable form:

$$
\frac{4 \stackrel{\circ}{\Lambda}}{d-1} h^{\mu \nu} \xi_{\nu}=2\left(\xi_{\nu} h_{\lambda}^{\nu}\right)^{[; \mu ; \lambda]}
$$

where $A^{[; \mu ; \lambda]} \equiv A^{; \mu ; \lambda}-A^{; \lambda ; \mu}$. In this form the current $J^{\mu}$ does not depend anymore on $\stackrel{\circ}{\Lambda}$ and $d$.

The following statement summarizes the mathematical achievement of this work: the skew-symmetric tensor

$$
\Omega^{\alpha \mu}=\frac{1}{64 \pi G}\left(Q_{[; \beta}^{\alpha \mu \beta \nu} \xi_{\nu]}-Q^{\alpha \mu \beta \nu} \xi_{\nu ; \beta}\right),
$$

where

$$
Q^{\alpha \mu \beta \nu} \equiv \gamma^{\mu \nu} h^{\alpha \beta}+\gamma^{\alpha \beta} h^{\mu \nu}-\gamma^{\alpha \nu} h^{\beta \mu}-\gamma^{\beta \mu} h^{\alpha \nu}
$$

satisfies

$$
J^{\mu}=2 \Omega_{; \alpha}^{\mu \alpha} .
$$

The tensor $Q^{\alpha \mu \beta \nu}$ has the same symmetries as the Riemman tensor. Defining the $(d-1)$-form

$$
\Omega=\frac{1}{(d-1) !} \Omega^{\mu \nu} \eta_{\mu \nu \alpha_{1} \ldots \alpha_{d-1}} d x^{\alpha_{1}} \wedge \ldots \wedge d x^{\alpha_{d-1}}
$$


then Eq. (3.9) is equivalent to

$$
J=d \Omega,
$$

which proves that $J$ is not only closed, but globally exact. Hence, the conserved quantities will be given by

$$
Q=\int_{\partial \Sigma} \Omega
$$

This total conserved quantity is gauge independent because it was reduced to an integral at the boundary at infinity. There, the true gauge tranformations (2.26) reduce to the identity because we are imposing the preservation of the asymptotic structure of the fields. Also, with the $(d-1)$-form $\Omega$ we do not need the knowledge of the gravitational field on the whole $\Sigma$ but only its asymptotic behaviour on $\partial \Sigma$, which makes the calculation of the total conserved quantities much easier and general.

Note that $\Omega$ does not depend neither on $d$ nor on $\Lambda$. All dependence on these parameters are contained in $h^{\mu \nu}$. However, we have to fix the background metric in some way because once a metric $g_{\mu \nu}$ is given, the determination of $h^{\mu \nu}$ will depend on the choice of $\gamma_{\mu \nu}$, as can be seen from Eq. (2.1). Note that even for asymptotically AdS spacetimes one could take Ricci flat background geometries just by making $\stackrel{\circ}{\Lambda}=0$ in Eq. (2.3): our results are independent on the choice of $\stackrel{\circ}{\Lambda}$. Let us then calculate the total energy of a simple asymptotically AdS spacetime, the Schwarzschild AdS solution in four dimensions, using a Ricci flat background, namely, a flat background. The Schwarzschild AdS solution is:

$$
d s^{2}=-\left[1+\left(\frac{r}{R}\right)^{2}-\frac{2 m}{r}\right] d t^{2}+\left[1+\left(\frac{r}{R}\right)^{2}-\frac{2 m}{r}\right]^{-1} d r^{2}+r^{2}\left[d \theta^{2}+\sin ^{2}(\theta) d \phi^{2}\right]
$$

where $R$ is the radius of curvature of such space, related to the effective cosmological constant $\Lambda_{\text {eff }}=\Lambda+8 \pi G \rho_{V}$ by $R=\left(-3 / \Lambda_{e f f}\right)^{1 / 2}$ ( $\rho_{V}$ is the matter vacuum energy density). The flat background metric is taken in spherical coordinates. The non null $h^{\mu \nu}$ are

$$
h^{t t}=\frac{r \rho^{2}-2 m}{r-2 m+r \rho^{2}},
$$

and

$$
h^{r r}=\frac{-2 m}{r}+\rho^{2}
$$

where $\rho \equiv r / R$. To calculate the total energy, we will take the timelike Killing vector field of flat spacetime, $\xi^{\mu}=\delta_{t}^{\mu}$. When we calculate the total energy using Eqs. (3.7), (3.8), (3.10) and (3.12), we obtain (from now on we will make $G=1)$ :

$$
E=\lim _{r \rightarrow \infty}\left[\frac{r^{2}\left(m+r \rho^{2}\right)}{2\left(r-2 m+r \rho^{2}\right)^{2}}+\frac{m}{2}-\frac{r^{3}}{R^{2}}\right]=\frac{m}{2}-\lim _{r \rightarrow \infty} \frac{r^{3}}{R^{2}}
$$

The last term is the energy of the anti-de Sitter solution, which is the Schwarzschild AdS solution with $m=0$. Subtracting it we find:

$$
E_{r e n}=\frac{m}{2}
$$

This result does not give the Schwarzschild mass, first calculated in the field theoretical approach in Ref. [9], when we put $\Lambda_{\text {eff }}$ to zero after the calculation. For the usual asymptotically flat Schwarzschild solution, the energy in Eq. (3.16), setting $\Lambda_{e f f}=\rho=0$ from the beginning, reads $E=m$. Hence, we have an internal inconsistency: the total energy of the Schwarzschild AdS solution using a flat background does not yield the total energy of the asymptotically

\footnotetext{
${ }^{1}$ In Ref. 10] another energy-momentum tensor is defined for flat background, and Eq. (2.18) is written in another form, with the presence of extra non linear terms in its left-hand-side. However, as total conserved quantities are calculated by means of asymptotic boundary integrals, only the linear terms given by $\Omega$ in Eq. (3.12) are important.
} 
flat Schwarzschild solution (calculated within the same rules) when we put $\Lambda_{\text {eff }}$ to zero. However, as we will see in the following, if we take an ADS background (with the same $\Lambda_{\text {eff }}$ ) in the calculation of the energy of the Schwarzschild AdS solution, the energy in the limit $\Lambda_{e f f}=0$ yields the energy of the asymptotically flat Schwarzschild solution calculated in Ref. [9]. This shows that the background metric is not arbitrary but is dictated by the asymptotic structure of the spacetime in question. If we take other simple examples, we can readily conclude that the background metric for asymptotic AdS spacetimes must not only satisfy $\stackrel{\circ}{R}_{\mu \nu}=2 \stackrel{\circ}{\Lambda} \gamma_{\mu \nu} /(d-1)$, with $\stackrel{\circ}{\Lambda}=\Lambda_{e f f}=\Lambda+8 \pi G \rho_{V}$, but it must also be asymptotically AdS. A metric which is asymptotically AdS, satisfies Eq. (2.3), and is regular everywhere must be the AdS metric (an analogous reasoning can be used for asymptotically flat spacetimes). Hence, the asymptotic structure, together with regularity assumptions, fixes the background metric. Also, the background AdS metric must be in the same coordinate system as the AdS asymptotic geometry at infinity, but this coordinate system may be arbitrary because $\Omega^{\alpha \mu}$ in Eq. (3.7) is a true tensor. This is equivalent to the imposition that only true gauge transformations which reduce to the identity at the spatial infinity are allowed. With these restrictions dictated by the asymptotic structure of the geometry under study, the conserved quantity (3.12) has no ambiguities: it is invariant under the allowed true gauge transformations, and the background metric is fixed by the asymptotic behaviour of the geometry.

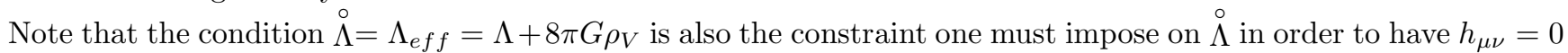
as a solution of the vacuum field equations (2.8).

With these rules in mind, let us calculate some conserved quantities for asymptotically AdS solutions. In the examples below we will take for convenience, and without loss of generality, that $\rho_{V}=0$ and hence $\stackrel{\circ}{\Lambda}=\Lambda$. Also, all the quantities calculated below are conserved because in all cases $\int_{B} J=0$, where $B$ is the timelike portion of $\partial M$ in Eq. (3.3), from where Eq. (3.4) follows.

\section{The Kerr-anti-de Sitter spacetime.}

The coordinates of the effective geometry $g_{\mu \nu}$ are such that it tends asymptotically to the anti-de Sitter metric in the form

$$
d^{\circ} s^{2}=-\left[1+\left(\frac{r}{R}\right)^{2}\right] d t^{2}+\left[1+\left(\frac{r}{R}\right)^{2}\right]^{-1} d r^{2}+r^{2}\left[d \theta^{2}+\sin ^{2}(\theta) d \phi^{2}\right]
$$

where, as before, $R=(-3 / \Lambda)^{1 / 2}$. In this case, the leading order aymptotic components of the gravitational field $h^{\mu \nu}$ read $\llbracket$

$$
\left\{\begin{aligned}
h^{t t} & =-\frac{2 m R^{4}}{r^{5}}\left[1-\alpha^{2} \sin ^{2} \theta\right]^{-5 / 2} \\
h^{t \phi} & =-\frac{2 a m R^{2}}{r^{5}}\left[1-\alpha^{2} \sin ^{2} \theta\right]^{-5 / 2} \\
h^{\phi \phi} & =-\frac{2 m a^{2}}{r^{5}}\left[1-\alpha^{2} \sin ^{2} \theta\right]^{-5 / 2} \\
h^{r r} & =-\frac{2 m}{r}\left[1-\alpha^{2} \sin ^{2} \theta\right]^{-3 / 2} \\
h^{r \theta} & =\frac{2 m a^{2}}{r^{4}}\left[1-\alpha^{2} \sin ^{2} \theta\right]^{-5 / 2} \sin \theta \cos \theta \\
h^{\theta \theta} & =-\frac{2 m a^{4}}{r^{7}}\left[1-\alpha^{2} \sin ^{2} \theta\right]^{-7 / 2} \sin ^{2} \theta \cos ^{2} \theta
\end{aligned}\right.
$$

where $\alpha=a / R$, and $a$ is related to the angular momentum per unit mass of the Kerr-anti-de Sitter spacetime. For the calculation of the gravitational energy, we take the timelike Killing vector field of the AdS spacetime $\xi^{\mu}=\delta_{t}^{\mu}$, which is also a Killing vector field of the effective geometry, and insert it in Eq. (3.7). The only nonzero component of $\Omega^{\alpha \mu}$ is $\Omega^{t r}$, which yields for $Q$ in Eq. (3.12)

$$
Q=E=\frac{m}{\left(1-\alpha^{2}\right)^{2}}
$$

agreeing with the results using pseudotensors [3], the hamiltonian formalism [4], and the quasilocal stress tensor [5]. Note that the flat space limit $\Lambda=0$ in the above expression yields the energy of the asymptotically flat Kerr spacetime calculated within the same rules. There are no internal inconsistencies.

The Kerr-anti-de Sitter spacetime also has a conserved angular momentum. To calculate it, we now have to take the Killing vector field $\xi^{\mu}=\delta_{\phi}^{\mu}$, which is again a Killing vector field of the effective geometry. We proceed in an analogous way obtaining

$$
Q=L_{\phi}=-\frac{m a}{\left(1-\alpha^{2}\right)^{2}}
$$

which coincide with calculations on Refs. 田, 5] 


\section{The Schwarzschild-anti-de Sitter spacetime in five dimensions.}

The Schwarzschild-anti-de Sitter metric in five dimensions reads

$$
\begin{aligned}
d s^{2}= & -\left[1+\left(\frac{r}{R}\right)^{2}-\left(\frac{r_{0}}{r}\right)^{2}\right] d t^{2}+\left[1+\left(\frac{r}{R}\right)^{2}-\left(\frac{r_{0}}{r}\right)^{2}\right]^{-1} d r^{2} \\
& +r^{2}\left\{d \theta_{1}^{2}+\sin ^{2}\left(\theta_{1}\right)\left[d \theta_{2}^{2}+\sin ^{2}\left(\theta_{2}\right) d \phi^{2}\right]\right\}
\end{aligned}
$$

where $R=(-6 / \Lambda)^{1 / 2}$, and the background metric is obtained from Eq. (3.22) by making $r_{0}=0$ in it. The non null $h^{\mu \nu}$ are

$$
h^{t t}=-\frac{l^{2}}{\left(1+\rho^{2}\right)\left(1+\rho^{2}-l^{2}\right)},
$$

and

$$
h^{r r}=l^{2},
$$

where $l \equiv r_{0} / r$ and, as before, $\rho \equiv r / R$. Taking again the timelike Killing vector field of the AdS spacetime $\xi^{\mu}=\delta_{t}^{\mu}$, and inserting it in Eq. (3.7), we obtain for the total the gravitational energy of this spacetime the value

$$
Q=E=\frac{3 \pi r_{0}^{2}}{8}
$$

which agree with the result of Ref. [12]. This is the only non null conserved quantity of this geometry.

\section{The near-horizon limit of the D3-brane}

The effective five-dimensional metric now reads

$$
d s^{2}=\left(\frac{r}{R}\right)^{2}\left\{-\left[1-\left(\frac{r_{0}}{r}\right)^{4}\right] d t^{2}+\left(d x^{i}\right)^{2}\right\}+\left[1-\left(\frac{r_{0}}{r}\right)^{4}\right]^{-1}\left(\frac{r}{R}\right)^{2} d r^{2} .
$$

The background metric is obtained by making $r_{0}=0$ in Eq. (3.26). It is now written in a different coordinate system than in the precedent example but in accordance with the asymototic limit of (3.26). The non null $h^{\mu \nu}$ are

$$
h^{t t}=-\frac{l^{4}}{\rho^{2}\left(1-l^{4}\right)}
$$

and

$$
h^{r r}=l^{4} \rho^{2},
$$

where $l$ and $\rho$ are defined as above. Taking again the timelike Killing vector field of the AdS spacetime, the gravitational energy now reads

$$
Q=E=\frac{3 r_{0}^{4}}{16 \pi R^{5}} \int d^{3} x
$$

which agree with the result of Ref. [12]. This is the only non null conserved quantity of this geometry. 


\section{CONCLUSION}

In this paper we have extended the field theoretical approach to GR to the case where the background metric satisfies $\stackrel{\circ}{R}_{\mu \nu}=2 \gamma_{\mu \nu} \stackrel{\circ}{\Lambda} /(d-1)$. After that, we have obtained a $(d-1)$-form $\Omega$ which, when integrated on asymptotic $(d-1)$ dimensional surfaces, yields the values of total energies and angular momenta of asymptotically $(d+1)$-dimensional AdS or flat spacetimes. Although the dynamics of the effective geometry does not depend on the background metric we choose, the values of those total conserved quantities are strongly affected by the choice we make. As we have shown in the text, if we do not choose judiciously the background metric we may obtain preposterous results for the gravitational energy. Hence, the ambiguity in the choice of the background metric may be eliminated only by going beyond the equations of motion and examining further concepts, like the consistency of calculations of total energy and angular momenta. These considerations indicate, together with regularity conditions, what is the background metric one should adopt.

The calculations of total conserved quantities using $\Omega$ yield finite results, and are gauge independent once one does not violate asymptotic conditions. This is not true, however, for conserved quantities contained in finite regions of the background space. These calculations may suffer from gauge ambiguities because the effective geometry may be presented in many different coordinate systems with the same asymptotic limits, and hence, in finite regions, the true gauge transformations (2.26) are not trivial. It should be interesting to investigate under what subgroup of the true gauge transformations $(2.26)$ is the $(d-1)$-form $\Omega$ given in Eqs. (3.7) and (3.10) invariant.

Let us now compare the $(d-1)$-form $\Omega$ given in Eqs. 3.7.3.10) and its integral with the quasilocal stress tensor of Ref. [13], and the surface integrals of Ref. [4]. They have in common the presence of a background (reference) space which is fixed by the asymptotic behaviour of the effective geometry. However, for the $(d-1)$-form $\Omega$, the presence of the background space is much more important. Contrary to the other prescriptions, the surfaces where the integrals are performed are defined on the background space, and the Killing vector fields which are present in $\Omega$ generates isometries of the background, not of the effective geometry. This last fact does not mean that we can have more conserved quantities than the number of Killing vectors of the effective geometry. If we use some Killing vector field of the background geometry which does not describe an isometry of the effective geometry than the integral $\int_{B} J$, where $B$ is the timelike portion of $\partial M$ in Eq. (3.3), is not zero and Eq. (3.4) does not follow. The quantity $\int_{\Sigma} J$ is not conserved because there is a flux of $J$ through $B$.

The $(d-1)$-form $\Omega$ can be used to calculate conserved quantities or fluxes through the boundary $B$ for more involved effective geometries. The fact that $\Omega$ is derived from a theory which describes the gravitational field as a spin-two field propagating on a fixed background may be useful to understand some aspects of the correspondence of conformal field theory in an AdS boundary and gravitational theory in AdS spaces.

\section{ACKNOWLEDGEMENTS}

We would like to thank the Cosmology Group of CBPF for useful discussions, and CNPq of Brazil for financial support.

[1] J. M. Maldacena, Adv. Theor. Math. Phys. 2, 231 (1997).

[2] E. Witten, Adv. Theor. Math. Phys. 2, 253 (1998).

[3] L. F. Abbott and S. Deser, Nucl. Phys. B 195, 76 (1982).

[4] M. Henneaux and C. Teitelboim, Commun. Math. Phys. 98, 391 (1985).

[5] N. Pinto-Neto and I. Damião Soares, Phys. Rev. D 52, 5665 (1995).

[6] V. Balasubramanian and P. Kraus, hep-th/9902121.

[7] R. C. Myers, hep-th/9903203.

[8] S.Deser, GRG, 1, 9 (1970).

[9] L.P.Grishchuk, A.N. Petrov and A.D. Popova, Commun. Math. Phys. 94, 379 (1984).

[10] S. V. Babak and L.P.Grishchuk, gr-qc/9907027.

[11] R. R. Silva, J. Math. Phys., 39, 6206 (1998).

[12] G. T. Horowitz and R. C. Myers, Phys. Rev. D 59, 026005 (1999).

[13] J. D. Brown and J. W. York, Jr., Phys. Rev. D 47, 1407 (1993). 